\title{
THE BASIS THEOREM FOR VECTOR SPACES OVER RINGS
}

\section{J. EVERETT}

It is the purpose of this note to establish the following theorem :

Theorem. $A$ vector space $M=u_{1} K+\cdots+u_{m} K$ of $m$ basis elements over a ring $K=\{0, a, b, \cdots, 1\}$ with unit 1 has the property that every subspace $N>0$ possesses $a$ basis of $n \leqq m$ elements if and only if $K$ is a right principal-ideal-ring without zero-divisors.

That such a ring insures the basis condition for subspaces is well known $\left[3\right.$, p. 121]. ${ }^{1}$

Suppose now that every subspace $N>0$ has a basis of $n \leqq m$ elements. It has been shown $[2$, Theorem $(F)]$ that every right ideal $R>0$ of $K$ must then have a single generator: $R=r_{0} K$, where $r_{0} k=0$ implies $k=0$. Moreover, since every right ideal has a finite set of generators, the ascending chain condition must hold for right ideals of $K$ $[3$, p. 26]. It therefore suffices to prove the following two lemmas.

LEMMA 1. In a ring $K$ with unit 1 and ascending chain condition for right ideals, equations $a b=1, a c=0$ imply $c=0$.

If $c \neq 0$, the linear transformation $k \rightarrow a k, k \in K$, would be of type (iv) $\left[2\right.$, p. 313], that is, $K / K_{0} \cong K$, and $0<K_{0}<K_{1}<K_{2}<\cdots$, where $K_{i}$ is defined inductively as the set of all elements of $K$ mapped into elements of $K_{i-1}$. This contradicts the chain condition.

LEMMA 2. A ring $K$ with unit in which every right ideal $R>0$ is of the form $r_{0} K$, where $r_{0} k=0$ implies $k=0$, has no zero divisors.

Let $s c=0, s \neq 0$, and $s K=r_{0} K \neq 0$, where $r_{0} k=0$ implies $k=0$. We have $s=r_{0} a, r_{0}=s b=r_{0} \cdot a b, r_{0}(a b-1)=0$, and hence $a b=1$. Also, $s c=0=r_{0} a c$, and $a c=0$. Since Lemma 1 applies to $K, c=0$.

It should be noted that the result follows also from a result of Baer's [ 1 , Theorem 5 or Lemma 4] which states that in a ring with unit and weak maximal condition, $a b=1$ implies $b a=1$.

\section{BIBLIOGRAPHY}

1. R. Baer, Inverses and zero-divisors, Bull. Amer. Math. Soc. vol. 48 (1942) pp. $630-638$.

Presented to the Society, November 25, 1944; received by the editors February 14, 1945.

1 Numbers in brackets refer to the bibliography. 
2. C. J. Everett, Vector spaces over rings, Bull. Amer. Math. Soc. vol. 48 (1942) pp. 312-316.

3. B. L. van der Waerden, Moderne Algebra, vol. 2, 1st ed., Berlin, 1931.

UNIVERSITY OF WISCONSIN

\section{ON A CONSTRUCTION FOR DIVISION ALGEBRAS OF ORDER 16}

R. D. SCHAFER

It is not known whether there exist division algebras of order 16 (or greater) over the real number field $\Re$. In discussing the implications of this question in algebra and topology, A. A. Albert told the author that the well known Cayley-Dickson process ${ }^{1}$ does not yield a division algebra of order 16 over $\Re$ and suggested a modification of that process which might. It is the purpose of this note to show that, while Albert's construction can in no instance yield such an algebra over $\Re$, it does yield division algebras of order 16 over other fields, in particular the rational number field $R$.

Initially consider an arbitrary field $F$. Let $C$ be a Cayley-Dickson division algebra of order 8 over $F$. Define ${ }^{2}$ an algebra of order 16 over $F$ with elements $c=a+v b, z=x+v y(a, b, x, y$ in $C)$ and with multiplication given by

$$
c z=(a+v b)(x+v y)=(a x+g \cdot y b S)+v(a S \cdot y+x b)
$$

where $S$ is the involution $x \rightleftarrows x S=t(x)-x$ of $C$ and $g$ is some fixed element of $C$. The Cayley-Dickson process is of course the instance $g=\boldsymbol{\gamma}$ in $F$.

For $A$ to be a division algebra over $F$ the right multiplication ${ }^{1} R_{z}$ must be nonsingular for all $z \neq 0$ in $A$. Now

$$
R_{z}=\left(\begin{array}{cc}
R_{x} & S R_{y} \\
S L_{y} L_{0} & L_{x}
\end{array}\right)
$$

Received by the editors January 19, 1945, and, in revised form, March 19, 1945.

${ }^{1}$ See [1] and [2] for background and notations. Numbers in brackets refer to the references cited at the end of the paper.

2 We should remark that this modification of the Cayley-Dickson process does yield non-alternative division algebras of orders 4 and 8 over $\Re$ when applied to the algebras of complex numbers and real quaternions instead of to $C$. See R. H. Bruck, Some results in the theory of linear non-associative algebras, Trans. Amer. Math. Soc. vol. 56 (1944) pp. 141-199, Theorem 16C, Corollary 1, for a generalization. 